\title{
Propiedades psicométricas de un cuestionario para evaluar la percepción de los trabajadores de las políticas de recursos humanos y su compromiso organizacional Psychometric properties of a questionnaire to evaluate the perception of the employees regarding Human Resources policies and their organizational commitment
}

\author{
Santiago Ávila Vila \\ (D) https://orcid.org/0000-0002-6565-0958 \\ Universidad CEU San Pablo, España. \\ san.avila.ce@ceindo.ceu.es \\ Marcelo Pascual Faura \\ (D) https://orcid.org/0000-0001-8358-3915 \\ Universidad CEU San Pablo, España. \\ mpascualfaura@gmail.com \\ Recibido: 02-11-2018
}

\section{Resumen}

El objetivo de este trabajo es desarrollar una herramienta que permita la evaluación del nivel de Compromiso Organizacional de los trabajadores y de la Percepción que estos tienen de determinadas Políticas de Recursos Humanos que inciden en este tipo de compromiso, para lo que se ha diseñado y contrastado las propiedades psicométricas de un cuestionario, que permitirá implementar estrategias de Dirección de Personas dirigidas a aumentar el nivel de compromiso de los trabajadores. Cuestionario que constituido por una parte ad hoc (ítems sobre las percepciones de sus políticas), y otra adaptada (organizational commitment questionnaire -OCQ- de Porter), fue cumplimentado por 706 sujetos (580 hombres y 126 mujeres). Los análisis factoriales exploratorios y confirmatorios realizados muestran que el modelo que mejor se ajusta a los datos obtenidos es el compuesto por cinco factores: compromiso organizacional, estilo de dirección, comunicación corporativa y reconocimiento, capacitación profesional, y retribución. La consistencia interna, estimada mediante el coeficiente $\alpha$ de Cronbach, es adecuada tanto para el cuestionario total como para cada uno de los factores.

Palabras clave: compromiso organizacional, ecuaciones estructurales, evaluación, percepción de las políticas de recursos humanos, propiedades psicométricas.

\begin{abstract}
The objective of this work is developing a tool that allows evaluating the level of the Organizational Commitment that the employees have regarding Human Resources Policies that have impact in this kind of commitment, for this study, it has been evaluated the psychometric properties of a questionnaire that will enable the implementation of People Management strategies aimed to improving the commitment of the employees. The questionnaire contains an ad hoc part (items on the perception of their policies), and another adapted part (organizational commitment questionnaire OCQ- Porter) and was answered by 706 individuals ( 580 men and 126 women). The exploratory and confirmatory factor analyzes carried out show that the model that best fits the data obtained is a five-factor model: organizational commitment, management style, corporate communication and recognition, professional training and compensation. The internal consistency, estimated using the Cronbach's $\alpha$ coefficient, is adequate for both the total questionnaire, and each of the factors.
\end{abstract}

Key words: organizational commitment, structural equations, evaluation, perception of human resources policies, psychometric properties.

\section{Sumario}

1. Introducción | 2. Objetivo del estudio | 3. Método | 3.1. Participantes | 3.2. Instrumentos y variables | 3.3. Análisis de Datos | 4. Resultados | 4.1. Fiabilidad del cuestionario general. V-1 | 4.2. Estructura factorial| 5. Conclusiones | Referencias bibliográficas

Cómo citar este artículo

Ávila Vila, S. y Pascual Faura, M. (2019): "Propiedades psicométricas de un cuestionario para evaluar la percepción de los trabajadores de las políticas de recursos humanos y su compromiso organizacional", methaodos. revista de ciencias sociales, 7 (2): 165-183. http://dx.doi.org/10.17502/m.rcs.v7i2.258 


\section{Introducción}

En la actualidad, cualquier organización se ve empujada a participar en una revolución constante forzada por el saber. Saber, que se debiera plasmar como la expresión de un talento que se desea innovador y comprometido. Cada vez es mayor el esfuerzo con el que deben emplearse los directivos en su tarea de atraer, desarrollar, y retener, el talento necesario para mantener una ventaja competitiva en sus organizaciones (Mercurio, 2015).

La empresa, conseguida la innovación en forma de nuevos productos y servicios, finalizará impactando en una sociedad de la cual forma parte. Entornos estables y conservadores, serán retados por la incertidumbre, la ambigüedad, la globalización y el cambio constante, con lo que cualquier organización se verá obligada a analizar la forma en que se pueden potenciar las relaciones que se establecen entre sus políticas de dirección de personas y el compromiso organizacional (Allen y Meyer, 1990; Cohen, 2007; Morrow, 2011); compromiso que como vínculo psicológico que es (Ayala, 2007; Meyer y Herscovitch, 2001; Mowday et al., 1979; Scholarios y Marks, 2004), se traducirá en una fuerza de identificación y participación del individuo con su organización (Chinchilla, 2000; Meyer et al., 2006; O’Reilly y Chatman, 1986).

Organización a la que, inmersa en un proceso de mejora continua, no le basta con evaluar el grado de compromiso de sus empleados, también, necesita analizar cómo se perciben las políticas de dirección de personas por parte de los trabajadores. La satisfacción, el compromiso la motivación no son políticas de recursos humanos en sentido estricto, sino que están más en el ámbito de las consecuencias de las prácticas implantadas en la organización. De hecho, cuando se quieren gestionar estos temas, donde se tiene que incidir es en las políticas (Maella, 2010). Como consecuencia, se establece una relación en la que la persona, aportando su saber-hacer, y por tanto la parte del trato que le corresponde, deberá encontrar en su empresa la reciprocidad debida (Ávila, 2017); con lo que cada parte, además de centrarse en sus expectativas implícitas, mutuas y recíprocas -ajenas al contrato formal de la relación laboral--, también deberá hacerlo con su responsabilidad: el trabajador en su compromiso, y la empresa en sus políticas (Argyris, 1960; Levinson et al., 1962; Schein, 1965).

No obstante, "la organización, al igual que la otra parte de la relación, proporciona el contexto para la creación del contrato psicológico, pero ella misma a cambio no puede tener un contrato psicológico con sus miembros" (Rousseau, 1989: 126). De ahí que tanto directivos como mandos intermedios, en su condición de representantes de la organización, sean la otra parte del contrato de los empleados, ya que un colectivo solo puede comunicar y negociar a través de sus representantes (Tena, 2002).

Desde tal perspectiva, las palabras, escritos, deducciones y hechos que tienen lugar en las interacciones cotidianas, se concretan como señales creíbles de futuras intenciones. En términos generales, operan dos grupos de factores: los mensajes externos -las políticas de dirección de personas como mecanismo principal-, y las interpretaciones que de los mismos realiza cada individuo (Rousseau y WadeBenzoni, 1994).

De acuerdo con (Schein, 1965), cuando el empleador no atiende debidamente a las expectativas de los empleados, su compromiso y conducta extra-rol se resienten, derivándose reacciones que conducirán a un descenso de la confianza, del compromiso, y de la disminución del rendimiento en el puesto de trabajo. "Comprender las percepciones de los empleados de las obligaciones mutuas puede ser al menos tan importante como crear una relación contractual con un particular conjunto de cláusulas" (Robinson et al., 1994: 149).

A partir de lo expuesto, debemos plantearnos cómo influye el contexto organizacional y social en el compromiso de los empleados. Por ejemplo, qué hace posible que unos trabajadores se muestren con un bajo compromiso en su país de origen y en una actitud contraria en otro. Así, si extrapolamos la investigación realizada por Bernárdez con trabajadores emigrantes mexicanos a EEUU, al colectivo de emigrantes independientemente del país de destino, se concluye que un trabajador inmerso en un sistema social que no le estimula ni le ofrece perspectivas claras de progreso, que responde reduciendo su contribución, disminuyendo su esfuerzo, y concentrando su energía y recursos en emigrar a otro país, cambia de comportamiento, entregando compromiso y contribución sobresalientes, cuando, a pesar del desarraigo y de no hablar el idioma, se encuentra con expectativas claras de progreso económico y sistemas de trabajo más eficientes (Bernárdez, 2009). 
Desde tal perspectiva, es a la organización a la que le corresponde facilitar un contexto adecuado para que el trabajador se sienta comprometido. La empresa, seleccionado el personal que como tal se le supone, deberá enfocar sus esfuerzos en generar políticas que propicien el contexto adecuado para generar este compromiso con la organización.

Habitualmente, se pierde de vista el carácter sistémico de los problemas de desempeño, centrados en los resultados y en el comportamiento del trabajador (su antecedente más inmediato). Muchas veces se dejan de analizar las dificultades que afronta y que originan la pérdida de su compromiso inicial. Obligar a un empleado a convivir con un sistema lleno de dificultades implicará, en la mayoría de las ocasiones, un deterioro de su motivación, empuje y compromiso (Bernárdez, 2006; Rummler y Brache, 2013).

La organización no puede actuar directamente sobre el trabajador sino es a través de sus políticas de dirección de personas, mejorarlas, y sobre todo establecer planes de comunicación adecuados, supondrá que este no tenga que convivir en un entorno que le haga perder su compromiso organizacional.

Desde el punto de vista organizativo, la cuestión no es si podemos mejorar este o aquel aspecto del comportamiento, sino qué estrategias van a permitir que se alcancen los mejores resultados. La mayoría de las personas se preocupan por conseguir un buen desempeño laboral porque tienen capacidad, motivación, y compromiso. A excepción de un porcentaje reducido de individuos, generalmente la gente se preocupa mucho por cómo se desenvuelve en el trabajo y, por tanto, solo se debería considerar su capacidad y motivación como fuente de problemas cuando se hayan agotado las posibilidades de mejora en la información que recibe, las herramientas con las que se le dota, la adecuada alineación de incentivos y desempeño y la capacitación profesional que se le ofrece (Gilbert, 2007).

De ahí que, cuando se pretende ayudar a los empleados a incrementar su compromiso, efectividad y rendimiento hay que concentrarse en la mejora del entorno que la organización crea para ellos (Cuadro 1).

Cuadro 1. Mejora del desempeño

\begin{tabular}{cccc}
\hline Apoyo del entorno & Información & Instrumentos & Incentivos \\
& C & (2) & 3 \\
\hline \multirow{2}{*}{ Tipo de Comportamiento } & Conocimiento & Capacidad & Motivos \\
& 4 & 5 & 6 \\
\hline
\end{tabular}

Fuente: Gilbert (2007: 92).

Gilbert mantiene que la secuencia de la figura 1 es la más eficiente, y lo es no porque los motivos sean de menor trascendencia que la información, instrumentos, incentivos o conocimiento, sino porque, en la mayoría de las situaciones, ayudar a superar deficiencias en los aspectos señalados resulta menos costoso (a la vez que evita la pérdida de compromiso) que estimular la motivación del trabajador, y puede conseguir, indirectamente, el mismo efecto.

De ahí que conocer la percepción de los empleados sobre la forma en que estos perciben las políticas de dirección de personas, nos ayudará a entender cómo contribuyen estas a la mejora del compromiso organizacional. Políticas que serán percibidas a través de dos mediadores: el propio jefe, con su particular estilo directivo, y la alta dirección, concretada en el establecimiento de dichas políticas. Según investigaciones realizadas por diversos autores (Bernárdez, 2006; Liu, 2009; Meyer et al., 2002; Saá y García, 2001; Shore y Wayne, 1993), el compromiso organizacional es un antecedente de mejor desempeño y resultados, por lo que resultará imprescindible cuidarlo.

Propiciar un buen ambiente, rodeando a los mejores de una red interna de talento, también potenciará su expresión (Guthridge et al., 2008). Al medir el compromiso en una organización, sabremos de la eficacia de sus políticas, y de lo que cabe hacer en ellas para que mejore. En consecuencia, y según lo expuesto, se plasmará como un indicador de la forma en que se perciben las políticas de dirección de personas, a la vez que se concretará como un antecedente de mejores desempeños. 


\section{Objetivo del estudio}

El objetivo de este trabajo es desarrollar una herramienta que permita la evaluación del nivel de compromiso organizacional de los trabajadores, y de la percepción que estos tienen de determinadas políticas de Recursos Humanos implantadas en la empresa, de tal forma que pueda ser empleada en ulteriores investigaciones. Para lo cual, se ha diseñado y contrastado las propiedades psicométricas de un cuestionario, que permitirá implementar estrategias concretas de Dirección de Personas dirigidas a aumentar el nivel de compromiso de los trabajadores, en base a la mejora de la percepción que tienen de las mencionadas políticas, con la finalidad de incrementar los índices de productividad y satisfacción laboral; y, por tanto, reducir las tasas de absentismo y rotación de personal y, por ende, los elevados costes directos e indirectos que estos aspectos acarrean a la empresa.

Analizamos la fiabilidad y validez de la escala mediante los coeficientes alfa y el análisis factorial, para demostrar su consistencia interna y validez de constructo. A priori, se espera que el análisis factorial exploratorio (rotación oblimin) muestre una estructura de cinco factores acorde con la estructura del cuestionario diseñado, que esperamos sea ratificada posteriormente mediante análisis factorial confirmatorio.

Se pretende elaborar un instrumento que sea útil en el estudio del análisis de la influencia que tiene la percepción de los trabajadores de determinadas políticas de dirección de personas sobre su nivel de compromiso organizacional, a la vez que se presentan datos de su estructura factorial, fiabilidad y validez.

\section{Método}

\subsection{Participantes}

El cuestionario fue cumplimentado por 706 trabajadores de la empresa de seguridad Securitas, repartidos por toda la geografía española y con una edad media de 42 años, una máxima de 64 años, y una mínima de 20 años, en la que los mayores porcentajes se corresponden con el rango de $40-49$ años (36,12\%), seguido de 20-39 años (35,98\%), y del que supera los 50 años (27,90\%). La distribución por sexos es de 580 hombres (82,2\%) y 126 mujeres (17,8\%) (Tabla 1$)$.

Tabla 1. Distribución socio laboral de la muestra clasificada por sexos

\begin{tabular}{lcccc}
\hline FUNCIÓN EN LA EMPRESA & H & M & TOTAL & $\begin{array}{c}\text { Relación } \\
\text { H/M }\end{array}$ \\
\cline { 2 - 5 } Directivos & 28 & 9 & 37 & 3,1 \\
Mandos Intermedios & 71 & 11 & 82 & 6,5 \\
Personal sin Responsabilidad sobre terceros & 481 & 106 & 587 & 4,5 \\
TOTAL & 580 & 126 & 706 & 4,6 \\
\hline
\end{tabular}

Fuente: Elaboración propia.

Como puede apreciarse en la Tabla 1, cabe señalar la baja presencia de personal directivo femenino (circunstancia que se podría calificar de "clásica" en el sentido de lo que ha venido siendo habitual en el ámbito empresarial hasta un pasado reciente); y que a día de hoy persiste en determinadas organizaciones, y de forma más acentuada en las de seguridad, por ser herederas de unos inicios -década de los 70 y 80 del siglo pasado- en los que la presencia de cuadros directivos procedentes de las fuerzas armadas y de seguridad del estado era mayoritaria. Si bien ya no es así, puesto que la actual procedencia directiva ya no se corresponde con la extracción anterior, la disfunción anterior perdura; mostrándose también, todavía más acentuada, en mandos intermedios, cometido al que se llega por promoción, y en menor grado, pero aun de forma significativa, en el personal sin responsabilidad sobre terceros, lo que refleja la visión que se tiene de una actividad que se sigue percibiendo masculina (aunque cada vez en menor medida) y que dificulta la conciliación familiar. El cuestionario fue enviado por correo electrónico desde la empresa y cumplimentado de forma totalmente anónima y confidencial por los empleados durante el mes de mayo de 2017. 


\subsection{Instrumentos y variables}

El cuestionario empleado en la encuesta consta de dos partes (ver Cuadro 2). La primera (P-1) está enfocada a la obtención de información sobre la manera en que los trabajadores perciben las siguientes políticas de dirección de personas:

- Estilo de dirección. Relativa a la forma en que el empleado percibe el desempeño directivo del superior inmediato en cuanto a jefe.

- Información y comunicación. Que trata de determinar la valoración que hace el trabajador sobre la información y comunicación recibidas y lo adecuado de las mismas.

- Capacitación profesional. Centrada en averiguar cómo percibe el personal la formación continua recibida para su actualización y reciclaje permanente, y los medios y herramientas de trabajo que se ponen a su disposición.

- $\quad$ Retribución. Percepción que tiene el trabajador sobre su sistema de compensación salarial.

Y la segunda (P-2), en la cual se analiza el:

- Compromiso organizacional. Que evalúa el deseo de permanecer y de mantener altos niveles de esfuerzo en la organización, aceptando sus metas y valores. Puntuaciones altas del empleado concuerdan con actitudes positivas hacia la empresa, con la que se siente identificado e intenta mantenerse en ella. Puntuaciones bajas nos presentan un empleado falto de compromiso.

Cuadro 2. V-1 del Cuestionario

$(\mathrm{P}-1)$

\begin{tabular}{|c|c|}
\hline Ítem & Estilo de dirección \\
\hline 1. & Mi jefe, me informa regularmente de la misión, objetivos, y responsabilidades de mi actual puesto de trabajo. \\
\hline 2. & Conozco, a través de él, lo que se espera de mí, así como la forma en que se va a valorar mi trabajo. \\
\hline 3. & Me ha informado, en el trabajo, qué conductas y acciones se consideran adecuadas. \\
\hline 4. & Mi jefe es correcto en las formas. \\
\hline 5. & Cumple lo previamente acordado. \\
\hline 6. & Atiende y escucha toda propuesta o sugerencia que se le haga e intenta que se lleve a efecto. \\
\hline 7. & Es justo en sus acciones. \\
\hline 8. & Se implica en el desarrollo de las personas que trabajamos con él. \\
\hline \multirow[t]{2}{*}{9.} & Es ejemplo de conducta en la defensa de la ética de la empresa. \\
\hline & Información y comunicación \\
\hline 10. & Recibo información acerca de los planes a futuro de la empresa. \\
\hline 11. & Recibo información sobre su evolución. \\
\hline 12. & $\begin{array}{l}\text { La empresa anima a quienes trabajamos en ella a que se propongan mejoras que favorezcan el rendimiento y el } \\
\text { clima laboral. }\end{array}$ \\
\hline 13. & $\mathrm{Si}$, como consecuencia de una propuesta, se obtiene mejoría, se premia. \\
\hline \multirow[t]{2}{*}{14.} & La información fluye habitualmente por lo que no puede considerarse un hecho excepcional. \\
\hline & Capacitación profesional \\
\hline 15. & Mis "herramientas" de trabajo son apropiadas. \\
\hline 16. & Los procedimientos de trabajo son adecuados. \\
\hline 17. & Recibo formación técnica adecuada. \\
\hline 18. & $\begin{array}{l}\text { Recibo formación en habilidades de relación con los clientes, compañeros, colaboradores...(Resolución de conflictos, } \\
\text { negociación, inteligencia emocional...). }\end{array}$ \\
\hline 19. & El despacho, oficina o lugar de trabajo en el que realizo mi actividad, es acogedor y agradable. \\
\hline \multirow[t]{2}{*}{20.} & Se trabaja con planificación, evitando que la obsolescencia tecnológica afecte a mi trabajo. \\
\hline & Retribución \\
\hline 21. & Mi retribución es adecuada en comparación con la que reciben mis colegas de trabajo. \\
\hline 22. & Mi retribución es adecuada en comparación con la que en otras empresas del sector se da para un cometido similar. \\
\hline 23. & La relación entre lo que aporto a la organización y lo que recibo de ella me parece correcta. \\
\hline 24. & La empresa tiene establecidos unos criterios claros de promoción interna. \\
\hline 25. & La empresa tiene como norma recompensar a los que desempeñan su trabajo de forma excelente. \\
\hline
\end{tabular}




\section{$(\mathrm{P}-2)$}

Compromiso organizacional

26. Estoy dispuesto a realizar un gran esfuerzo para contribuir al éxito de la organización.

27. Cuando hablo con mis amigos les digo que mi organización es un gran lugar para trabajar.

28. Soy leal a esta organización.

29. Aceptaría casi cualquier tipo de tarea con tal de seguir trabajando aquí.

30. Considero que mis valores y los de la dirección de la organización son muy similares.

31. Me siento orgulloso de decir que formo parte de esta organización.

32. Si el tipo de trabajo en otra organización fuera similar preferiría continuar en la actual.

33. La dirección de la organización contribuye a que me sienta motivado en el desempeño de mi trabajo.

34. En mis circunstancias actuales, me afectaría bastante tener que abandonar esta organización.

5stoy encantado de haber escogido esta organización para trabajar y no otras a las que consideré unirme en su momento.

36. Sí que merece la pena permanecer trabajando en esta organización indefinidamente.

37. Casi nunca discrepo con la política de la organización en asuntos relacionados con los empleados.

38. Me importa mucho el futuro de esta organización

39. Para mi esta es la mejor organización posible para trabajar.

40. Definitivamente, la decisión de trabajar en esta organización ha sido un acierto por mi parte.

Fuente: P-1, Elaboración propia; P-2, Adaptado (Mowday et al., 1979: 228).

Cuestionario que, en su P-1, se plasma como una fuente de información que ha sido elaborada ad hoc para el presente estudio; siendo la P-2, una versión traducida al español del OCQ de Porter (Mowday et al., 1979: 228), en la que se han adaptado los ítems de expresión negativa a otros que, manteniendo la misma intencionalidad, se presentan afirmativos - la dificultad que suponía invertir la forma de calificar recomendaba hacerlo así-. Ambas partes se presentan en una escala de respuestas tipo Likert que va desde totalmente en desacuerdo (1) a totalmente de acuerdo (5). La estructura del cuestionario en esta primera versión (V-1) está formada por 40 ítems, de los cuales los 25 primeros corresponden a la parte diseñada ad hoc, y los quince últimos a la adaptada del OCQ de Porter.

\subsection{Análisis de Datos}

Se realizaron análisis factoriales exploratorios para el estudio de la dimensionalidad del cuestionario, así como un análisis factorial de segundo orden como base de propuesta para un análisis factorial confirmatorio. En primer lugar, se calculó el coeficiente alfa de Cronbach del cuestionario total así como el de cada una de sus subescalas, para a continuación proceder a realizar un análisis factorial exploratorio con el método de rotación oblicua tipo Oblimin, con la finalidad de clarificar la estructura de los factores que se extrajeron del cuestionario; posteriormente, se realizó un análisis factorial de segundo orden para ver cómo se modifica la estructura, y que sirvió de base para el confirmatorio con el fin de reafirmarse en la estructura encontrada. Por último, se volvió a calcular el coeficiente de fiabilidad, tanto del cuestionario final como el de cada uno de los factores que se extrajeron con los análisis factoriales, mediante el alfa de Cronbach.

Para esta primera parte el paquete estadístico utilizado fue el SPSS_24. De su resultado, se siguió un análisis factorial confirmatorio con empleo del paquete estadístico SPSS Amos 25. Para evaluar la bondad de ajuste del modelo se utilizó: el índice $\chi 2$ (chi cuadrado) /DF (en AMOS: CMIN/DF), en el que con el fin de reducir la dependencia de $\chi 2$ con el tamaño de la muestra se divide entre los grados de libertad, siendo aceptables los valores incluidos en el rango 2:1 o 3:1 (Ruiz et al., 2010); el índice RMSEA (Aproximación de la raíz cuadrada del error), que, aunque propuesto hace tiempo por (Steiger y Lind, 1980), solo recientemente ha sido reconocido como uno de los índices que ofrece una mayor información sobre las estructuras de covarianza, en donde valores inferiores a 0,08 indican un buen ajuste (Bollen y Stine, 1993; Littlewood y Bernal, 2014); el índice GFI (Índice de bondad de ajuste), que es una medida de la proporción de varianzacovarianza observada que puede ser explicada por la varianza-covarianza reproducida por el modelo, siendo recomendables valores superiores a 0,90 (Cupani, 2012; Escobedo et al., 2016); el índice PGFI (Índice de bondad parsimónico), que se diferencia del anterior en que está corregido en función de los grados de libertad del modelo; se considera que valores superiores a 0,6 indican buena parsimonia (Littlewood y Bernal, 2014). 


\section{Resultados}

\subsection{Fiabilidad del cuestionario general. V-1}

Se estudió la fiabilidad del cuestionario a través del coeficiente alfa de Cronbach, obteniéndose un valor de 0,971; indicador que se sitúa por encima del mínimo en investigación aplicada (0,90-0,95) (Nunnally, 1978), lo que permite confirmar una buena consistencia interna (George y Mallery, 2003). También, se calcularon los índices de fiabilidad de cada una de las subescalas, oscilando estos entre 0,83 y 0,96 lo que nos confirma su buena consistencia interna (Tabla 2).

Tabla 2. Alfa de Cronbach de cada subescala

\begin{tabular}{cccccc}
\hline Subescala & $\begin{array}{c}\text { Estilo } \\
\text { directivo }\end{array}$ & $\begin{array}{c}\text { Información y } \\
\text { comunicación }\end{array}$ & $\begin{array}{c}\text { Capacitación } \\
\text { profesional }\end{array}$ & $\begin{array}{c}\text { Retribución } \\
\text { Organizacional }\end{array}$ \\
\hline Alfa de Cronbach & 0,959 & 0,939 & 0,877 & 0,826 & 0,950 \\
\hline
\end{tabular}

Fuente: Elaboración propia a partir de tratamiento estadístico con SPSS_24.

En la Tabla 3, se presentan los principales estadísticos univariados para cada uno de los cuarenta ítems del cuestionario, así como la correlación elemento total corregida. En ella, se puede observar cómo las medias más altas se corresponden con la subescala compromiso organizacional, siendo la media más alta la obtenida en el ítem 28 (4,401), seguida de la de los ítems 26, 38 y 32, que también se sitúan por encima de cuatro. Sin embargo, las subescalas de información y comunicación junto con la de retribución, presentan las medias más bajas, siendo el ítem 13 el de menor puntuación $(2,055)$. Las variables presentan cierto grado de asimetría, siendo mayoría las asimétrico-negativas; cabe resaltar que la subescala de información y comunicación se presenta toda ella asimétrica-positiva.

Si observamos la media y mediana, vemos que en la mayor parte de los ítems son similares o casi iguales, lo que nos indica que las distribuciones son todas prácticamente normales; no obstante, y dado el valor que toma la mediana (5) para los ítems 28 y 38 , significar que el ítem 28, recibe una puntuación de 5 en 440 de los 706 casos posibles, lo que representa un 62,32\% del total, siendo, en el caso del ítem 38, de 357 , lo que supone un 50,57\% del total también.

En relación con el cumplimiento de la condición de normalidad de las variables de la escala, Curran, West, y Finch, (1996) fijan los límites, en valor absoluto, que permiten establecer si el comportamiento de una distribución se asemeja o no a una normal. Según dichos autores, valores comprendidos entre 0 y 2 para la asimetría, y entre 0 y 7 para la curtosis, informan de comportamientos semejantes a una distribución normal. De ahí que, consultada la tabla de descriptivos, se pueda comprobar que todas las variables presentan índices ajustados al comportamiento de una distribución normal.

Los índices de asimetría en valor absoluto de todas las variables se encuentran por debajo de 2: el caso más desfavorable es el ítem 28 con un valor de 1,71. No obstante, hay que resaltar que los valores más altos de la tabla se encuentran todos en la escala de compromiso organizacional, en particular, son los ítems 26 (1,40), 28 (1,71), y 38 (1,37); el resto, presenta índices por debajo o muy próximos a 1.

En cuanto a los índices de curtosis, todos los valores se encuentran por debajo de 7 , siendo, nuevamente, el caso más desfavorable el ítem 28 , con un valor de 2,80 . Hay que señalar que los valores más altos de la tabla se encuentran nuevamente en los mismos ítems de la escala de compromiso organizacional: $26(1,66)$; $28(2,80)$; y 38 (1,35), seguidos de dos del factor estilo de dirección: $2(1,35)$ y $8(1,34)$; el resto, presenta índices por debajo o muy próximos a 1.

Resaltar, como resumen, que de un total de 40 ítems y con un criterio mucho más restrictivo: 1 (Lloret-Segura et al., 2014), solo habría dos variables (26 y 28), de la escala de compromiso organizacional, que se presentan claramente por encima de dicho criterio $(26:+0,66$ y $28:+1,80)$, aunque siempre dentro de los valores inicialmente referidos. Ítems que recogen el sentir de la organización en la que se realiza el estudio, ya que es, sino la más importante, de las dos más importantes del sector en España, lo que favorece la existencia de un fuerte compromiso organizacional, puesto que toda expectativa de cambio de empresa, bien por propia iniciativa o por vía de la subrogación de personal, se concretará evocadora de una peor situación organizacional. 
Tabla 3. Estadísticos univariados y coeficiente de correlación elemento total corregida

\begin{tabular}{|c|c|c|c|c|c|c|c|}
\hline Subescala & Ítem & Media & Mediana & $\begin{array}{l}\text { Desviación } \\
\text { estándar }\end{array}$ & Asimetría & Curtosis & $\begin{array}{c}\text { Correlación elemento-total } \\
\text { corregida }\end{array}$ \\
\hline \multirow{9}{*}{ 1. Estilo de Dirección } & 1 & 2,956 & 3,000 & 1,417 & 0,015 & $-1,275$ & 0,722 \\
\hline & 2 & 2,806 & 3,000 & 1,452 & 0,140 & $-1,346$ & 0,753 \\
\hline & 3 & 3,323 & 3,000 & 1,378 & $-0,362$ & $-1,069$ & 0,654 \\
\hline & 4 & 3,654 & 4,000 & 1,336 & $-0,707$ & $-0,637$ & 0,654 \\
\hline & 5 & 3,370 & 4,000 & 1,414 & $-0,390$ & $-1,136$ & 0,728 \\
\hline & 6 & 3,218 & 3,000 & 1,393 & $-0,268$ & $-1,167$ & 0,732 \\
\hline & 7 & 3,180 & 3,000 & 1,364 & $-0,210$ & $-1,132$ & 0,756 \\
\hline & 8 & 3,038 & 3,000 & 1,442 & $-0,053$ & $-1,339$ & 0,776 \\
\hline & 9 & 3,248 & 3,000 & 1,433 & $-0,325$ & $-1,201$ & 0,762 \\
\hline \multirow{5}{*}{$\begin{array}{l}\text { 2. Información } \\
\text { Y Comunicación }\end{array}$} & 10 & 2,320 & 2,000 & 1,303 & 0,567 & $-0,856$ & 0,692 \\
\hline & 11 & 2,377 & 2,000 & 1,286 & 0,491 & $-0,920$ & 0,697 \\
\hline & 12 & 2,470 & 2,000 & 1,377 & 0,438 & $-1,097$ & 0,731 \\
\hline & 13 & 2,055 & 2,000 & 1,187 & 0,848 & $-0,225$ & 0,682 \\
\hline & 14 & 2,394 & 2,000 & 1,243 & 0,444 & $-0,864$ & 0,758 \\
\hline \multirow{6}{*}{$\begin{array}{l}\text { 3. Capacitación } \\
\text { profesional }\end{array}$} & 15 & 3,139 & 3,000 & 1,274 & $-0,241$ & $-0,989$ & 0,608 \\
\hline & 16 & 3,106 & 3,000 & 1,205 & $-0,210$ & $-0,891$ & 0,680 \\
\hline & 17 & 3,061 & 3,000 & 1,302 & $-0,136$ & $-1,040$ & 0,668 \\
\hline & 18 & 2,769 & 3,000 & 1,347 & 0,119 & $-1,172$ & 0,629 \\
\hline & 19 & 3,099 & 3,000 & 1,351 & $-0,184$ & $-1,132$ & 0,500 \\
\hline & 20 & 2,822 & 3,000 & 1,301 & 0,043 & $-1,089$ & 0,699 \\
\hline \multirow{5}{*}{ 4. Retribución } & 21 & 2,924 & 3,000 & 1,282 & $-0,039$ & $-1,008$ & 0,479 \\
\hline & 22 & 3,254 & 3,000 & 1,239 & $-0,279$ & $-0,771$ & 0,380 \\
\hline & 23 & 2,606 & 3,000 & 1,263 & 0,276 & $-0,971$ & 0,675 \\
\hline & 24 & 2,392 & 2,000 & 1,270 & 0,443 & $-0,901$ & 0,672 \\
\hline & 25 & 2,108 & 2,000 & 1,200 & 0,744 & $-0,483$ & 0,682 \\
\hline \multirow{15}{*}{$\begin{array}{l}\text { 5. Compromiso } \\
\text { organizacional }\end{array}$} & 26 & 4,178 & 4,000 & 1,023 & $-1,400$ & 1,664 & 0,523 \\
\hline & 27 & 3,525 & 4,000 & 1,257 & $-0,494$ & $-0,726$ & 0,747 \\
\hline & 28 & 4,401 & 5,000 & 0,927 & $-1,713$ & 2,800 & 0,513 \\
\hline & 29 & 2,868 & 3,000 & 1,324 & 0,056 & $-1,047$ & 0,506 \\
\hline & 30 & 3,208 & 3,000 & 1,308 & $-0,245$ & $-0,999$ & 0,743 \\
\hline & 31 & 3,756 & 4,000 & 1,290 & $-0,794$ & $-0,420$ & 0,747 \\
\hline & 32 & 4,016 & 4,000 & 1,170 & $-1,112$ & 0,487 & 0,642 \\
\hline & 33 & 2,761 & 3,000 & 1,355 & 0,152 & $-1,174$ & 0,819 \\
\hline & 34 & 3,755 & 4,000 & 1,291 & $-0,775$ & $-0,451$ & 0,575 \\
\hline & 35 & 3,799 & 4,000 & 1,198 & $-0,780$ & $-0,236$ & 0,724 \\
\hline & 36 & 3,824 & 4,000 & 1,242 & $-0,790$ & $-0,388$ & 0,712 \\
\hline & 37 & 3,159 & 3,000 & 1,272 & $-0,150$ & $-0,928$ & 0,618 \\
\hline & 38 & 4,163 & 5,000 & 1,077 & $-1,365$ & 1,345 & 0,561 \\
\hline & 39 & 3,469 & 4,000 & 1,284 & $-0,506$ & $-0,720$ & 0,669 \\
\hline & 40 & 3,824 & 4,000 & 1,172 & $-0,806$ & $-0,127$ & 0,724 \\
\hline
\end{tabular}

Fuente: Elaboración propia a partir de tratamiento estadístico con SPSS_24.

La correlación elemento-total corregida, siendo siempre positiva, se presenta con pesos superiores a 0,50 en todos los ítems con excepción de las variables 21 y 22, que muestran pesos respectivos de 0,48 y 0,38 . No obstante, al presentar una carga superior a 0,35, valor a partir del cual una correlación elementototal corregida se considera estadísticamente significativa más allá del 1\% (Cohen y Manion, 1990), se decidió su inclusión; lo que indica que todos ellos contribuyen a medir lo que mide el cuestionario y además en la misma dirección. 


\subsection{Estructura factorial}

La idoneidad de poder realizar los análisis factoriales se confirmó calculando el índice Kaiser-Meyer-Olkin y la prueba de esfericidad de Barlett $(\mathrm{KMO}=0,969 ;$ Chi-cuadrado $=25491,345 ; \mathrm{gl}=780 ;$ Sig. $=0,000)$. Mediante el análisis factorial exploratorio se extrajeron cinco factores, utilizando como método de extracción el de componentes principales, que siguiendo la regla de Kaiser (1958) mostraron un valor propio mayor que uno, y de rotación oblicua tipo Oblimin. Se ha utilizado este método al suponer que los factores están relacionados entre sí (Ferrando y Anguiano-Carrasco, 2010). De este modo, se pudieron identificar, a partir de la matriz de componentes, cinco claros y diferenciados factores, que daban una explicación de la varianza acumulada del 69,308\%; porcentaje comprendido en el rango (60\% - 70\%) que se considera adecuado dentro del ámbito de las ciencias sociales (Barbero et al., 2013).

Tabla 4. Matriz de factores rotados, valores propios y varianza explicada. Rotación Oblimin

\begin{tabular}{|c|c|c|c|c|c|}
\hline \multirow{2}{*}{ Ítems } & \multicolumn{5}{|c|}{ Componente } \\
\hline & ED & $\mathrm{CO}$ & CCR & RE & $\mathrm{CP}$ \\
\hline 1 & 0,700 & & & & \\
\hline 2 & 0,665 & & & & \\
\hline 3 & 0,675 & & & & \\
\hline 4 & 0,881 & & & & \\
\hline 5 & 0,849 & & & & \\
\hline 6 & 0,839 & & & & \\
\hline 7 & 0,895 & & & & \\
\hline 8 & 0,817 & & & & \\
\hline 9 & 0,780 & & & & \\
\hline 10 & & & 0,816 & & \\
\hline 11 & & & 0,844 & & \\
\hline 12 & & & 0,767 & & \\
\hline 13 & & & 0,733 & & \\
\hline 14 & & & 0,667 & & \\
\hline 15 & & & & & $-0,818$ \\
\hline 16 & & & & & $-0,762$ \\
\hline 17 & & & & & $-0,604$ \\
\hline 18 & & & & & $-0,553$ \\
\hline 19 & & & & & $-0,773$ \\
\hline 20 & & & & & $-0,670$ \\
\hline 21 & & & & 0,863 & \\
\hline 22 & & & & 0,901 & \\
\hline 23 & & & & 0,564 & \\
\hline 24 & & & 0,515 & & \\
\hline 25 & & & 0,569 & & \\
\hline 26 & & 0,752 & & & \\
\hline 27 & & 0,664 & & & \\
\hline 28 & & 0,799 & & & \\
\hline 29 & & 0,606 & & & \\
\hline 30 & & 0,482 & & & \\
\hline 31 & & 0,810 & & & \\
\hline 32 & & 0,745 & & & \\
\hline 33 & & & 0,362 & & \\
\hline 34 & & 0,695 & & & \\
\hline 35 & & 0,785 & & & \\
\hline 36 & & 0,820 & & & \\
\hline 37 & & 0,481 & & & \\
\hline 38 & & 0,877 & & & \\
\hline 39 & & 0,821 & & & \\
\hline 40 & & 0,850 & & & \\
\hline Valores propios: & 19,081 & 3,785 & 2,047 & 1,551 & 1,259 \\
\hline Varianza explicada (\%) & 47,703 & 9,463 & 5,116 & 3,878 & 3,148 \\
\hline Acumulada (\%) & 47,703 & 57,166 & 62,282 & 66,160 & 69,308 \\
\hline
\end{tabular}

Fuente: Elaboración propia a partir de tratamiento estadístico con SPSS_24. 
La Tabla 4 muestra la matriz de factores rotados, valores propios, y varianza explicada por cada factor y acumulada. En ella, y a pesar de utilizar la rotación oblicua, se puede observar que ningún ítem presenta saturaciones superiores a 0,40 en ningún otro factor que no sea en el de carga.

Como podemos observar, el primer factor está integrado por nueve ítems que se corresponden con la subescala del cuestionario que hemos denominado estilo de dirección, y que hacen referencia a un estilo de dirección participativo y de reconocimiento del trabajo bien hecho. Por tanto, mantendremos la denominación de este factor como "Estilo de dirección (ED)".

El segundo factor está formado por catorce ítems, coincidentes todos ellos con el cuestionario OCQ de Porter a excepción del 33, que pasa a presentar su saturación más alta en el factor tres. Por tanto, mantendremos la denominación de este factor como "Compromiso organizacional (CO)".

El tercer factor está formado por ocho ítems, de los cuales los cinco que presentan los pesos más altos corresponden a la subescala que habíamos denominado información y comunicación, los siguientes dos ítems con puntuaciones más altas son, el 25 con un peso de 0,57, y el 24 con un peso de 0,52, que en el cuestionario inicial estaban en la subescala que habíamos denominado retribución, y que ahora pasan a presentar sus saturaciones más elevadas en este factor tres. Por último, el ítem 33, que inicialmente estaba situado en la escala de compromiso, y que como ya hemos comentado no presenta saturaciones en ella y sí en este factor tres, con un peso de 0,36. Por tanto, y a la vista de estos resultados, pasamos a denominar este factor como "Comunicación corporativa y reconocimiento (CCR)", ya que tres de los ocho ítems $(12,13$, 25) hacen referencia al valor empresarial del reconocimiento de tanto las propuestas de mejora, que favorezcan un buen clima y rendimiento, como del trabajo bien hecho.

El cuarto factor está formados por tres ítems, el 22 con un peso de 0,90, el 21 con un peso de 0,86, y el 23 con un peso de 0,56, y que inicialmente estaban entre los cinco ítems que formaban la subescala sistema de retribución, denominación que seguimos manteniendo para este factor; ya que los tres ítems que lo definen hacen referencia a los tres requisitos básicos que tradicionalmente tiene que cumplir un sistema retributivo: que sea internamente equitativo, externamente competitivo, y motivador para el trabajador. Se le denominó "Retribución (RE)".

Por último, el quinto factor queda constituido por seis ítems, que se corresponden con los seis de la subescala que habíamos denominado capacitación profesional, en la cual se hace referencia a las herramientas y procedimientos de trabajo, así como a la formación continua tanto técnica como tecnológica como al desarrollo de habilidades sociales necesarias para el puesto de trabajo, y, por último, al entorno del trabajo. Por tanto, mantenemos el nombre de este factor como "capacitación profesional continua en su sentido más amplio, abarcando diversas áreas de conocimiento (CP)".

Tras este primer análisis, se realizó un análisis factorial de segundo orden con el objeto de simplificar y clarificar la estructura derivada del primer análisis factorial, y comprender mejor los agrupamientos obtenidos.

El análisis factorial de segundo orden arroja cuatro factores, que explican el $100 \%$ de la varianza (véase la Tabla 5, que muestra la matriz de factores rotados de $2^{\circ}$ orden por el método de rotación Oblimin, así como la varianza explicada por cada uno de los factores); coincidentes todos con los hallados en el de primer orden a excepción del factor $C P$, cuyos ítems pasan a repartir sus saturaciones en los factores CCR (ítems 17, 18 y 20), RE (ítems 16 y 19) y ED (ítem 15). Desde una perspectiva lógica resulta razonable que los ítems 17 y 18 saturen en CCR, puesto que la formación que reciben los trabajadores, bien técnica o en habilidades sociales, forma parte de la comunicación, como que también sature el ítem 20, ya que cuando la empresa trabaja con planificación, y evita la obsolescencia técnica, favorece el trabajo bien hecho, y su reconocimiento.

En relación con los ítems 16 y 19, que tratan sobre lo adecuado de los procedimientos, despacho, oficina o lugar de trabajo, resulta lógico que saturen en $\mathrm{RE}$, ya que es un factor que informa de condiciones que pueden llegar a ser motivadoras para el trabajador, como sería disponer de unas instalaciones y procesos adecuados.

Por último, el ítem 15, que informa sobre lo apropiado de los medios con que se dota al personal, carga sobre el factor ED; las herramientas de trabajo, al resultar un bien necesario para la ejecución de la tarea, suponen un motivo de relación habitual con el jefe respectivo, es por ello por lo que su estilo de dirección influirá en la percepción que tenga el trabajador de la forma en que se atienden sus necesidades de disponer de los medios oportunos. 
Tabla 5. Matriz de factores rotados de $2^{\circ}$ orden. Varianza explicada por cada factor. Rotación Oblimin

\begin{tabular}{|c|c|c|c|c|}
\hline \multirow{2}{*}{ Ítems } & \multicolumn{4}{|c|}{ Componente } \\
\hline & $\mathrm{CO}$ & CCR & ED & RE \\
\hline 1 & & & 0,936 & \\
\hline 2 & & & 0,917 & \\
\hline 3 & & & 0,930 & \\
\hline 4 & & & 0,932 & \\
\hline 5 & & & 0,949 & \\
\hline 6 & & & 0,982 & \\
\hline 7 & & & 0,971 & \\
\hline 8 & & & 0,987 & \\
\hline 9 & & & 0,993 & \\
\hline 10 & & 0,950 & & \\
\hline 11 & & 0,946 & & \\
\hline 12 & & 0,977 & & \\
\hline 13 & & 0,954 & & \\
\hline 14 & & 0,982 & & \\
\hline 15 & & & 0,629 & \\
\hline 16 & & & & 0,659 \\
\hline 17 & & 0,726 & & \\
\hline 18 & & 0,831 & & \\
\hline 19 & & & & 0,727 \\
\hline 20 & & 0,711 & & \\
\hline 21 & & & & 0,862 \\
\hline 22 & & & & 0,837 \\
\hline 23 & & & & 0,979 \\
\hline 24 & & 0,957 & & \\
\hline 25 & & 0,930 & & \\
\hline 26 & 0,931 & & & \\
\hline 27 & 0,997 & & & \\
\hline 28 & 0,960 & & & \\
\hline 29 & 0,973 & & & \\
\hline 30 & 0,916 & & & \\
\hline 31 & 0,998 & & & \\
\hline 32 & 0,976 & & & \\
\hline 33 & & 0,796 & & \\
\hline 34 & 0,977 & & & \\
\hline 35 & 0,979 & & & \\
\hline 36 & 0,983 & & & \\
\hline 37 & 0,972 & & & \\
\hline 38 & 0,953 & & & \\
\hline 39 & 0,979 & & & \\
\hline 40 & 0,989 & & & \\
\hline Varianza explicada (\%) & 37,96 & 29,35 & 21,47 & 11,22 \\
\hline Acumulada (\%) & 37,96 & 67,31 & 88,78 & 100 \\
\hline
\end{tabular}

Fuente: Elaboración propia a partir de tratamiento estadístico con SPSS_24.

Tomando como base los resultados obtenidos en los análisis factoriales previos, primer y segundo orden, se procedió al análisis factorial confirmatorio mediante el método de mínimos cuadrados generalizados, que presenta las mismas propiedades que el de máxima verosimilitud en condiciones de normalidad multivariante menos rigurosas y que resulta más adecuado cuando se incrementa el tamaño de la muestra (Lévy y Varela, 2006; Littlewood y Bernal, 2014).

Siendo el peso mínimo aceptable de un indicador en relación a su constructo, de una carga factorial de 0,70, (Littlewood y Bernal, 2014) - de esa forma se elimina al máximo la colinealidad entre variables-, se suprimieron aquellos ítems que en el submodelo de medida no llegaron a alcanzar dicha carga. 
Así, en el factor CO se eliminaron siete ítems: 29, 30, 31, 32, 34, 35, 37. La información del ítem 31 - me siento orgulloso de decir que formo parte de esta organización-, quedaría recogida por el 27 -cuando hablo con mis amigos les digo que mi organización es un gran lugar para trabajar-.

La de los ítems 29, 32 y 35 -aceptaría casi cualquier tipo de tarea con tal de seguir trabajando aquí; si el tipo de trabajo en otra organización fuera similar preferiría continuar en la actual; y estoy encantado de haber escogido esta organización para trabajar y no otras a las que consideré unirme en su momento-, por el 40 - definitivamente, la decisión de trabajar en esta organización ha sido un acierto por mi parte-.

La del 34 -en mis circunstancias actuales, me afectaría bastante tener que abandonar esta organización-, por el 36 y 38 -sí que merece la pena permanecer trabajando en esta organización indefinidamente; me importa mucho el futuro de esta organización-.

La de los ítems 30 y 37 -considero que mis valores y los de la dirección de la organización son muy similares; casi nunca discrepo con la política de la organización en asuntos relacionados con los empleados-, por el 26, 28 y 39 -estoy dispuesto a realizar un gran esfuerzo para contribuir al éxito de la organización; soy leal a esta organización; para mi esta es la mejor organización posible para trabajar-.

En el factor CCR se eliminaron dos ítems: 24 y 25. La información de ambos ítems -la empresa tiene establecidos unos criterios claros de promoción interna; la empresa tiene como norma recompensar a los que desempeñan su trabajo de forma excelente-, quedaría recogida por el 12 y 13 -la empresa anima a quienes trabajamos en ella a que se propongan mejoras que favorezcan el rendimiento y el clima laboral; si como consecuencia de una propuesta, se obtiene mejoría, se premia-.

Por último, en el factor CP se eliminaron tres ítems: 16, 18 y 19. La información de los ítems 16 y 19 - los procedimientos de trabajo son adecuados; el despacho, oficina o lugar de trabajo en el que realizo mi actividad, es acogedor y agradable-, quedaría asumida por el 15 -mis herramientas de trabajo son apropiadas-.

La del ítem 18 -recibo formación en habilidades de relación con los clientes, compañeros, colaboradores...-, quedaría recogida por el 17 -recibo formación técnica adecuada-. En los factores ED y RE no se suprimió ningún ítem.

Se contrastaron dos modelos diferentes: el modelo 1, que en coincidencia con el resultado del análisis factorial de segundo orden, está formado por cuatro factores (CO, CCR, ED, RE), y el modelo 2 que, en sintonía con el resultado del análisis factorial exploratorio de primer orden, propone una estructura de cinco factores (ED, CO, CCR, RE, CP).

Posteriormente, y atendiendo a los índices de modificación, se reconfigura el modelo 2, con un cambio de saturación del ítem 33 (23 en Cuadro 3), que pasa de saturar en CCR a CP, además de relacionar los errores de los ítems 7, 8 y 9 (14, 15 y 16 en Cuadro 3). Relación que encuentra fundamento en que "los errores entre dos ítems redundantes no pueden ser independientes, ya que... las respuestas siguen estando relacionadas debido a la semejanza de contenidos" (Ferrando y Anguiano-Carrasco, 2010: 25); tal es el caso de los ítems citados: 7. Es justo en sus acciones; 8 . Se implica en el desarrollo de las personas que trabajamos con él; y 9. Es ejemplo de conducta en la defensa de la ética de la empresa. En donde los valores de ejemplaridad, justicia e implicación se manifiestan redundantes en sus respectivos enunciados. En la Tabla 6 se presentan los índices de ajuste de los modelos.

Tabla 6. Índice de bondad de ajuste de los dos modelos propuestos

\begin{tabular}{lcccccc}
\hline Modelo Factorial & \multicolumn{7}{c}{ RMSEA } \\
\cline { 2 - 7 } Modelo 1. Cuatro factores. & 3,590 & 0,057 & 0,061 & 0,064 & 0,875 & 0,741 \\
Modelo 2. Cinco factores. & 3,279 & 0,053 & 0,057 & 0,061 & 0,887 & 0,743 \\
Modelo 2 Modificado. & 2,789 & 0,047 & 0,050 & 0,054 & 0,905 & 0,751 \\
\hline
\end{tabular}

Fuente. Elaboración propia.

El "modelo 2 modificado" es el que presenta los mejores índices, lo que indica un ajuste y mejora significativos con respecto a los modelos 1 y 2 en todos sus índices y dentro del rango de valores que se consideran adecuados. Quedando el modelo propuesto como se muestra en el Cuadro 3, en la que se puede observar su solución estandarizada. 


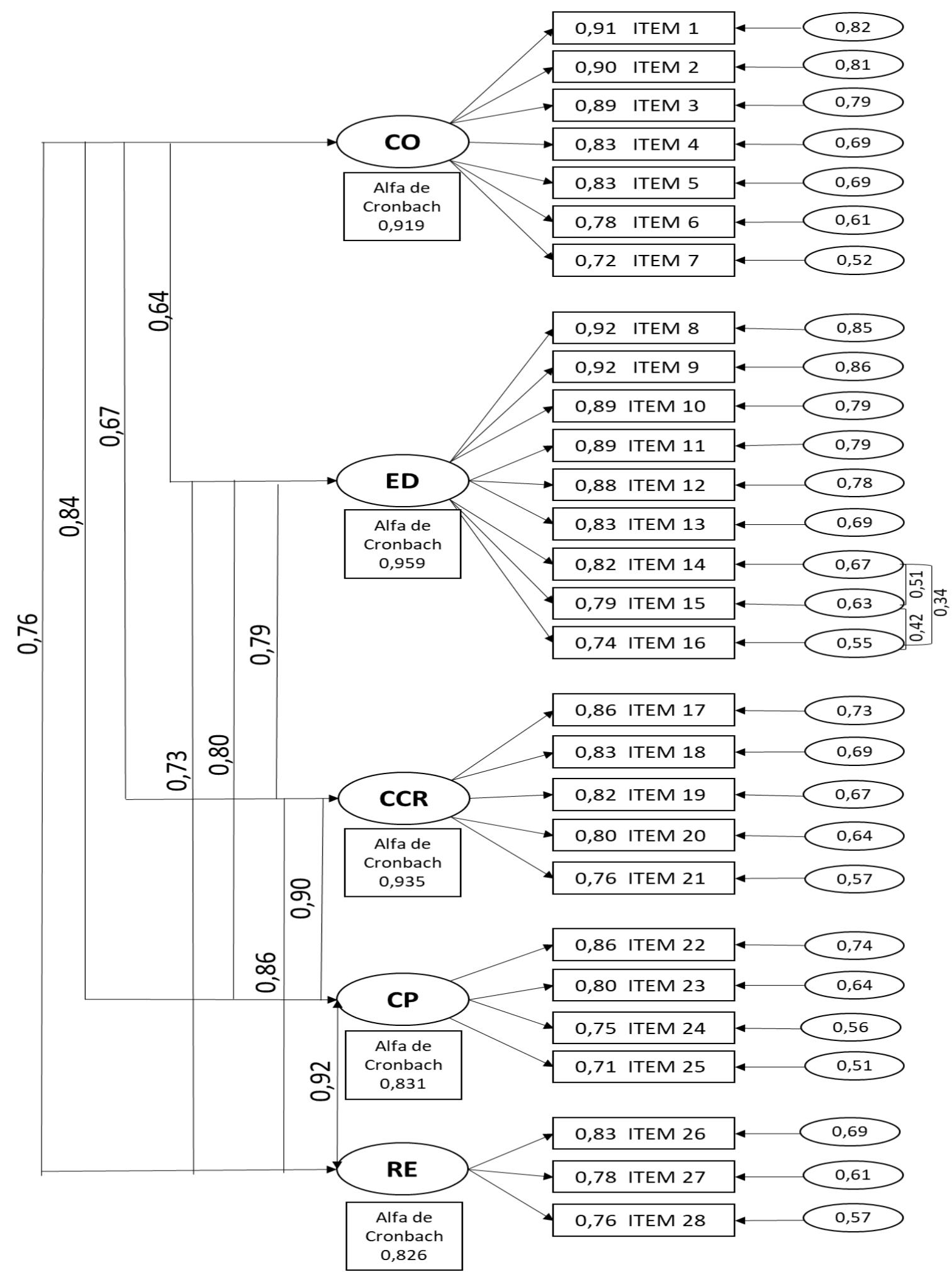

Fuente: Elaboración propia a partir de tratamiento estadístico con SSPS Amos 25. 
La Tabla 7. muestra que las cargas factoriales se sitúan entre 0,72 y 0,91 en el factor CO; 0,74 y 0,92 en ED; 0,76 y 0,86 en CCR; 0,71 y 0,86 en CP; y entre 0,76 y 0,83 en RE. En cuanto a las correlaciones entre factores, son todas significativas al 1\%; siendo las del compromiso con el resto de los factores de: 0,84 con $\mathrm{CP} ; 0,76$ con $\mathrm{RE} ; 0,67$ con $\mathrm{CCR}$; y 0,64 con ED. El resto de los factores correlacionan entre sí con un valor máximo de 0,92 entre CP y RE, y un valor mínimo de 0,73 entre RE y ED.

Tabla 7. Solución estandarizada de M2 modificado

\begin{tabular}{|c|c|c|c|c|c|}
\hline \multirow{2}{*}{ Ítems } & \multicolumn{5}{|c|}{ Componente } \\
\hline & $\mathrm{CO}$ & ED & CCR & $\mathrm{CP}$ & RE \\
\hline 1 & 0,91 & & & & \\
\hline 2 & 0,90 & & & & \\
\hline 3 & 0,89 & & & & \\
\hline 4 & 0,83 & & & & \\
\hline 5 & 0,83 & & & & \\
\hline 6 & 0,78 & & & & \\
\hline 7 & 0,72 & & & & \\
\hline 8 & & 0,92 & & & \\
\hline 9 & & 0,92 & & & \\
\hline 10 & & 0,89 & & & \\
\hline 11 & & 0,89 & & & \\
\hline 12 & & 0,88 & & & \\
\hline 13 & & 0,83 & & & \\
\hline 14 & & 0,82 & & & \\
\hline 15 & & 0,79 & & & \\
\hline 16 & & 0,74 & & & \\
\hline 17 & & & 0,86 & & \\
\hline 18 & & & 0,83 & & \\
\hline 19 & & & 0,82 & & \\
\hline 20 & & & 0,80 & & \\
\hline 21 & & & 0,76 & & \\
\hline 22 & & & & 0,86 & \\
\hline 23 & & & & 0,80 & \\
\hline 24 & & & & 0,75 & \\
\hline 25 & & & & 0,71 & \\
\hline 26 & & & & & 0,83 \\
\hline 27 & & & & & 0,78 \\
\hline 28 & & & & & 0,76 \\
\hline ED & $0,64^{* *}$ & & & & \\
\hline CCR & $0,67^{\text {** }}$ & $0,79 * *$ & & & \\
\hline $\mathrm{CP}$ & $0,84^{* *}$ & 0,80 ** & $0,90 * *$ & & \\
\hline RE & $0,76^{* *}$ & $0,73^{* *}$ & $0,86^{* *}$ & $0,92^{\star *}$ & \\
\hline
\end{tabular}

Fuente: Elaboración propia a partir de tratamiento estadístico con SSPS Amos 25

** Correlación significativa al 0,01

Conviene resaltar la congruencia del análisis factorial de segundo orden, en el que se informa que el factor CP es subsumido por el resto de los factores; tres de sus ítems: 17, 18, y 20 pasan a saturar en CCR; dos: 16 y 19 en RE; y el 15 en ED, con las correlaciones encontradas en el análisis factorial confirmatorio posterior, puesto que CP correlaciona con CCR: 0,90 y con RE: 0,92; haciéndolo en menor medida con ED: 0,80 .

También, se examinó la fiabilidad de V2 adaptado $(0,962)$, así como la de cada uno de sus cinco factores; coeficientes que se sitúan entre 0,83 de RE y CP, y 0,96 de ED, confirmándose con ello su alta consistencia interna tanto en los factores como en el total del cuestionario.

Estos resultados avalan empíricamente la estructura propuesta, quedando la V-2 del cuestionario formada por los siguientes factores e ítems ordenados de mayor a menor por su carga factorial (Cuadro 4). Tabla que muestra la estructura del cuestionario final, así como el alfa de cada factor y del cuestionario total. 
Cuadro 4. V-2 del cuestionario. Cuestionario final

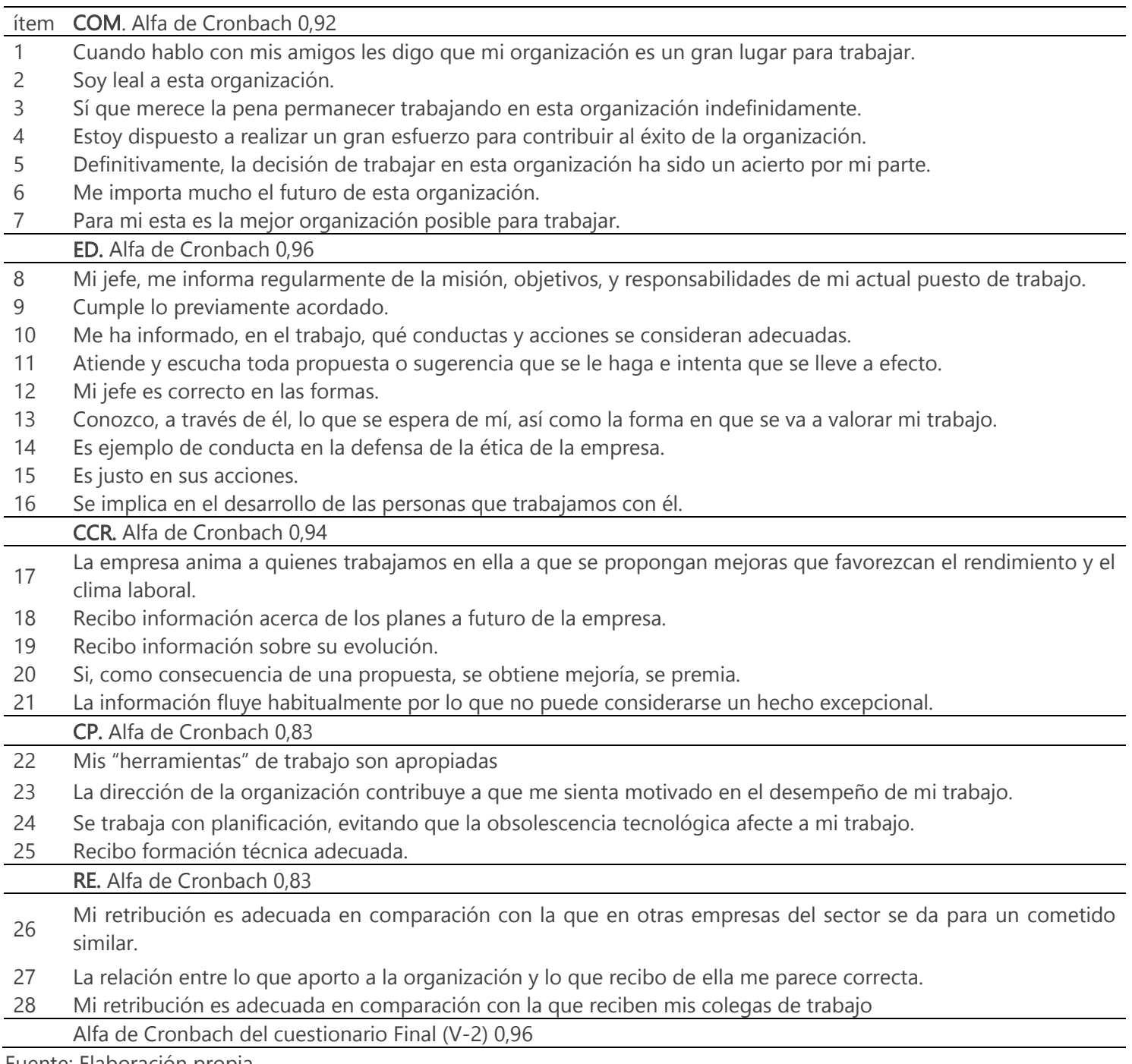
Fuente: Elaboración propia.

\section{Conclusiones}

El objetivo de este estudio era diseñar un cuestionario que permitiera, en investigaciones posteriores, evaluar la percepción que tienen los empleados sobre determinadas políticas de dirección de personas hipotéticamente relacionadas con el compromiso organizacional, compromiso que se concretaría como la consecuencia de una perspectiva psicológica, que entiende que los comportamientos extra-rol se derivan de un vínculo emocional que impulsa a hacer el mayor esfuerzo posible por realizar un buen trabajo (Chinchilla, 2000; Mathieu y M. Zajac, 1990; Mowday et al., 1979; O'Reilly y Chatman, 1986; Varona, 1993).

Disponer de un instrumento de estas características, fiable y válido, es de suma importancia de cara a obtener datos relativos del impacto que tienen determinadas políticas de dirección de personas sobre el compromiso organizacional de los empleados de la organización, para en su caso, establecer estrategias concretas que permitan mejorarlo. Como resultado de los análisis factoriales exploratorio y confirmatorio, se propone un modelo de cinco factores: 
- Compromiso organizacional, que se refiere a la actitud proactiva que toma el individuo en mantener altos niveles de esfuerzo en la organización junto con el deseo de permanecer en ella, aceptando sus metas y valores.

- Estilo de dirección, que recoge la forma en que los empleados perciben el estilo directivo del jefe inmediato, y que hace referencia no solo a un tipo de dirección participativo y a su implicación con el desarrollo de las personas de su equipo, sino que también pretende retroalimentar continuamente las conductas que se esperan del empleado en su trabajo. Es decir, un estilo de dirección que cree espacios de confianza, responsabilidad, y participación, que faciliten la mayor aportación posible de los miembros de un equipo.

- Comunicación corporativa y reconocimiento, que trata sobre la forma en que el trabajador percibe que se atienden sus necesidades de información y comunicación, así como de reconocimiento por el trabajo bien hecho.

- Capacitación profesional, que recoge el modo en que el personal percibe la formación continua recibida tanto técnica como tecnológica como de habilidades sociales necesarias para un mejor desempeño en el puesto de trabajo, así como de las herramientas que se ponen a su disposición y de los procedimientos a seguir.

- Retribución, que trata sobre lo que tradicionalmente se espera de un sistema retributivo: que sea equitativo internamente y competitivo externamente además de que resulte motivador para el trabajador.

El Modelo hipotetizado a través de los diversos análisis realizados tanto exploratorios como confirmatorios, determina que el compromiso organizacional vendría relacionado con la capacitación profesional, la retribución, la comunicación corporativa y reconocimiento, y el estilo de dirección. Esta nueva estructura presenta un mayor potencial explicativo que el que ofrecía la perspectiva lógica de la que se partía para elaborar el cuestionario inicial.

El conocimiento de las variables que pueden incidir en mejorar el grado o nivel de compromiso organizacional de los trabajadores es primordial para los responsables de la dirección de personas en las empresas, a la hora de diseñar e implantar estrategias de recursos humanos tendentes a reforzar los niveles de compromiso en aras de incrementar los niveles de productividad, mejorar la satisfacción laboral, retener el talento, y, por tanto, reducir las tasas de rotación externa y absentismo laboral, con la consecuente reducción de los altos costes directos e indirectos que estos aspectos conllevan para las empresas.

Para este conocimiento, los modelos de ecuaciones estructurales aportan la metodología adecuada para aislar variables relevantes y determinar su influencia dentro de los modelos teóricos sobre el fenómeno de estudio. De acuerdo con Barbero et al.:

Aun cuando los modelos de ecuaciones estructurales proporcionan una excelente metodología para el estudio de relaciones de causalidad, y, por tanto, para la obtención de evidencias empíricas para la manipulación de variables con ciertas garantías de éxito, no cabe plantearse que las variables independientes de los modelos que tienen un buen ajuste provoquen causalidad. A lo sumo, podemos llegar a concluir que las relaciones que hipotetiza el investigador se encuentran presentes en los datos analizado (2007: 420-421).

Con este objetivo, se estudió si la percepción que tienen los trabajadores de una empresa acerca de las siguientes políticas de dirección de personas: estilo de dirección (ED), comunicación corporativa y reconocimiento $(\mathrm{CCR})$, retribución $(\mathrm{RE})$ y capacitación profesional $(\mathrm{CP})$, estaban relacionadas con su grado de compromiso organizacional (CO).

En términos generales, se ha encontrado que efectivamente estas variables se relacionan con el nivel de compromiso organizacional (CO), ya que como puede observarse en la Tabla 7, todas ellas presentan correlaciones con un nivel de significación de un alfa $\leq 1 \%$. Por tanto, podemos considerar que si establecemos estrategias de dirección de recursos humanos que refuercen la percepción que tienen los trabajadores de la empresa sobre las mencionadas políticas, directamente estaremos mejorando su grado de compromiso organizacional. 
En conclusión, el cuestionario que aquí se propone parece un buen instrumento para evaluar la percepción de los trabajadores de las políticas de dirección de personas que inciden en su compromiso organizacional. Por este motivo, se propone como una herramienta de evidente utilidad en futuras investigaciones sobre este problema, de cara a planificar y diseñar las políticas que hagan posible que el trabajador se concrete en comportamientos que vayan mucho más allá de lo tenido por estricta obligación.

\section{Referencias bibliográficas}

Allen, N. y Meyer, J. (1990): "The Measurement and Antecedents of Affective, Continuance and Normative Commitment to the Organization." Journal of Occupational Psychology, 63(1): 1-18. http://dx.doi.org/ 10.1111/j.2044-8325.1990.tb00506.x

Argyris, C. (1960): Understanding Organizational Behavior. Homewood: Dorsey Press.

Ávila, S. (2017): Desde la felicidad hacia el compromiso. Madrid: Pearson Eduación.

Ayala, J. (2007): Conocimiento, innovación y emprendedores: camino al futuro. Logroño: Universidad de la Rioja.

Barbero, M., Holgado, F., Vila, E. y Chacón, S. (2007): "Actitudes, hábitos de estudio y rendimiento en matemáticas", Psicothema, 19(3): 413-421. Disponible en web: http://www.psicothema.com/pdf/3379.pdf.

—, Vila, E. y Holgado, F. (2013): Introducción básica al análisis factorial. Madrid: UNED.

Bernárdez, M. (2006): Tecnología del desempeño humano. Bloomington: Global Business Press.

- (2009): Desempeño humano. Manual de consultoría. Volumen 1. Bloomington: Global Business Press.

Bollen, K. y Stine, R. (1993): Testing Estructural Equation Models. Newbury Park: Sage. Disponible en web: http://linkinghub.elsevier.com/retrieve/pii/S0123592316300420.

Chinchilla, N. (2000): Ties of Belonging and Organizational Commitment. Barcelona: IESE Business School. [Nota técnica].

Cohen, A. (2007): "Commitment before and after: An Evaluation and Reconceptualization of Organizational Commitment", Human Resource Management Review, 17(3): 336-354. https://doi.org/10.1016/j.hrmr.2007.05.001

Cohen, L. y Manion. L. (1990): Métodos de investigación educativa. Madrid: La Muralla.

Cupani, M. (2012): "Análisis de ecuaciones estructurales: conceptos, etapas de desarrollo y un ejemplo de aplicación", Tesis, 1: 186-199. Disponible en web: https://revistas.unc.edu.ar/index.php/tesis/article/view/2884.

Curran, P. J., West, S. J. y Finch, J. F. (1996): "The Robustness of Test Statistics to Nonnormality and Specification Error in Confirmatory Factor Analysis", Psychological Methods, 1(1): 16-29. http://dx.doi.org/10.1037/1082-989X.1.1.16

Escobedo, M. T., Hernández-Gomez, J., Estebané, V. y Martínez, G. (2016): "Modelos de ecuaciones estructurales: características, fases, construcción, aplicación y resultados", Ciencia \& Trabajo, 18(55): 1622. http://dx.doi.org/10.4067/S0718-24492016000100004

Ferrando, P. y Anguiano-Carrasco, C. (2010): "El análisis factorial como técnica de investigación en psicología", Papeles del Psicologo, 31(1): 18-33.

George, D. y Mallery, P. (2003): SPSS for Windows Step by Step: A Simple Guide and Reference. 11.0. 4a. Boston: Allyn and Bacon. https://doi.org/10.4324/9781351033909

Gilbert, T. (2007): Human Competence: Engineering Worthy Performance. San Francisco: Pfeiffer.

Guthridge, M., Komm, A. B. y Lawson, E. (2008): "Making Talent a Strategic Priority", McKinsey Quarterly, 1(1): 48-59. Disponible en web: https://www.veruspartners.net/wp-content/uploads/old_articles/mata08.pdf.

Kaiser, H. (1958): "The Varimax Criterion For Analytic Rotation In Factor Analysis", Psychometrika, 23(3): 187200. http://dx.doi.org/10.1007/BF02289233

Levinson, H., Price, C., Munden, K. y Solley, C. (1962): Men, Management, and Mental Health. Cambridge: Harvard University.

Lévy, J. P. y Varela, J. (2006): Modelización con estructuras de covarianzas en Ciencias Sociales: temas esenciales, avanzados y aportaciones especiales. Oleiros (La Coruña): Netbiblo.

Littlewood, H. F. y Bernal, E. R.. (2014): Mi primer modelamiento de ecuaciones estructurales. Naucalpan: Herman Frank Littlewood Zimmerman. [2 ${ }^{\mathrm{a}} \mathrm{Ed}$.]

Liu, Y. (2009): "Perceived Organizational Support and Expatriate Organizational Citizenship Behavior: The 
Mediating Role of Affective Commitment towards the Parent Company", Personnel Review, 38: 3. https://doi.org/10.1108/00483480910943359

Lloret-Segura, S., Ferreres-Traver, A., Hernández-Baeza, A. y Tomás-Marco, I. (2014): "El análisis factorial exploratorio de los ítem: una guía práctica, revisada y actualizada", Anales de Psicología, 30(3): 11511169. http://dx.doi.org/10.6018/analesps.30.3.199361

Maella, P. (2010): ¿Cómo establecer una estrategia de Recursos Humanos eficaz? Barcelona: IESE Business School. [Occasional Paper].

Mathieu, J. y Zajac, D. (1990): "A Review and Meta-Analysis of the Antecedents, Correlates, and Consequences of Organizational Commitment", Psychological Bulletin, 108(2): 171-194. http://dx.doi.org/10.1037/00332909.108.2.171

Mercurio, Z. (2015): "Affective Commitment as a Core Essence of Organizational Commitment: An Integrative Literature Review", Human Resource Development Review, 14(4): 389-414. https://doi.org/10.1177/1534484315603612

Meyer, J. y Herscovitch, L. (2001): "Commitment in the Workplace: Toward a General Model", Human Resource Management Review, 11(3): 299-326. https://doi.org/10.1016/S1053-4822(00)00053-X

- Becker, T. y Van Dick, R. (2006): "Social Identities and Commitments at Work; toward an Integrative Model", Journal of Organizational Behavior, 27(5): 665-683. https://doi.org/10.1002/job.383

—, Stanley, D., Herscovitch, L. y Topolnytsky, L. (2002): "Affective, Continuance, and Normative Commitment to the Organization: A Meta-Analysis of Antecedents, Correlates, and Consequences", Journal of Vocational Behavior, 61(1): 20-52. https://doi.org/10.1006/jvbe.2001.1842

Morrow, P. (2011): "Managing Organizational Commitment: Insights from Longitudinal Research", Journal of Vocational Behavior, 79(1): 18-35. https://doi.org/10.1016/j.jvb.2010.12.008

Mowday, R., Steers, R. y Porter, L. (1979): "The Measurement of Organizational Commitment", Vocational Behavior, 14(2): 224-247. https://doi.org/10.1016/0001-8791(79)90072-1

Nunnally, J. (1978): Psychometric Theory. Ann Arbor, Michigan: McGraw-Hill. [2a Ed.].

O'Reilly, C. y Chatman, J. (1986): "Organizational Commitment and Psychological Attachment: The Effects of Compliance, Identification, and Internalization on Prosocial Behavior", Journal of Applied Psychology, 71(3): 492-499. http://dx.doi.org/10.1037/0021-9010.71.3.492

Robinson, S., Kraatz, M. y Rousseau. D. (1994): "Changing Obligations and the Psychological Contract: A Longitudinal Study", Academy of Management Journal, 37(1): 137-152. http://dx.doi.org/10.2307/256773

Rousseau, D. (1989): "Psychological and Implied Contracts in Organisations", Employee Responsibilities and Rights Journal, 2(2): 121-139. http://dx.doi.org/10.1007/BF01384942

- y Wade-Benzoni, K. (1994): "Linking Strategy and Human Resource Practices: How Employee and Customer Contracts Are Created", Human Resource Management, 33(3): 463-489. https://doi.org/10.1002/hrm.3930330312

Ruiz, M. A., Pardo, A. y San Martín, R. (2010): "Modelo de Escuaciones Estructurales", Papeles Del Psicólogo, 31(1): 34-45. Disponible en web: www.redalyc.org/articulo.oa?id=77812441004.

Rummler, G. y Brache, A. (2013): Improving Performance: How to Manage the White Space on the Organization Chart. San Francisco: Jossey-Bass. [3 Ed.].

Saá, P. y García, J. M. (2001): "El Sistema de Recursos Humanos y el desarrollo de capacidades organizativas: una aplicación empírica en el sector de las Cajas de Ahorros españolas". Disponible en web: http://dialnet.unirioja.es/servlet/articulo?codigo=195495\&info=resumen\&idioma=ENG.

Schein, E. (1965): Organizational Psychology. Englewood Cliff, NJ: Prentice-Hall.

Scholarios, D. y Marks, A. (2004): "Work-life Balance and the Software Worker", Human Resource Management Journal, 14(2): 54-74. https://doi.org/10.1111/j.1748-8583.2004.tb00119.x

Shore, L. y Wayne, S. (1993): "Commitment and Employee Behavior: Comparison of Affective Commitment and Continuance Commitment With Perceived Organizational Support", Journal of Applied Psychology, 78(5): 774-780. http://dx.doi.org/10.1037/0021-9010.78.5.774

Steiger, J. y Lind, J. (1980): Statistically Based Tests for the Number of Common Factors. lowa.

Tena, G. (2002): "El contrato psicológico: relación empresa-trabajador", Acciones e Investigaciones Sociales, 15: 85-107. Disponible en web: https://dialnet.unirioja.es/servlet/articulo?codigo=284117.

Varona, F. (1993): "Conceptualización y supervisión de la comunicación y el compromiso organizacional", Diálogos de La Comunicación, 35: 69-77. Disponible en web: http://dialogosfelafacs.net/wpcontent/uploads/2015/35/35-revista-dialogos-conceptualizacion-y-supervision-de-la-comunicacion.pdf. 
Breve CV de los autores:

Santiago Ávila Vila es Doctor en Economía por la Universidad CEU San Pablo, Escuela Internacional de Doctorado (CEINDO). Ejerce la docencia como Profesor Adjunto en el Departamento de Organización y Dirección de Personas de EAE Business School. MBA por el IESE. Es autor de varios libros de comportamiento organizacional de la Editorial Pearson.

Marcelo Pascual Faura es Doctor en Psicología por la Universidad Complutense de Madrid, Profesor Adjunto de la Universidad CEU San Pablo. Ejerce la docencia en el área de Recursos Humanos en el Grado en Administración y Dirección de Empresas. Los campos de investigación sobre los que trabaja son: gestión del compromiso organizacional, satisfacción laboral, comunicación interna y reestructuración empresarial. 